\title{
A recurrence for bounds on dominating sets in $k$-majority tournaments
}

\author{
Dror Fidler \\ dror.fid@gmail.com \\ Submitted: Dec 3, 2010; Accepted: Aug 7, 2011; Published: Aug 19, 2011 \\ Mathematics Subject Classification: 05C20, 05C69, 91B14
}

\begin{abstract}
A $k$-majority tournament is realized by $2 k-1$ linear orders on the set of vertices, where a vertex $u$ dominates $v$ if $u$ precedes $v$ in at least $k$ of the orders. Various properties of such tournaments have been studied, among them the problem of finding the size of a smallest dominating set. It is known that 2-majority tournaments are dominated by 3 vertices and that $k$-majority tournaments are dominated by $O(k \log k)$ vertices. However, precise values are not known even for $k=3$. We establish new upper bounds for the size of a smallest dominating set in $k$-majority tournaments that considerably improve upon previous bounds for small $k$. In particular our result shows that 3-majority tournaments are dominated by at most 12 vertices.
\end{abstract}

\section{Introduction}

A $k$-majority tournament is a finite tournament, $T=(V, E)$, which is realized by $2 k-1$ linear orders (lists) on the set of vertices. For every two vertices $u, v \in V,(u, v) \in E$ if and only if the index of $u$ is smaller than the index of $v$ in at least $k$ of the lists. $k$-majority tournaments arise in social choice theory, where each vertex may represent a candidate, and the lists represent the ranking of the candidates by different voters.

The connection between $k$-majority tournaments and general tournaments has been explored. The function $k_{0}(n)$ denotes the least integer $k$, such that every tournament with $n$ vertices may be represented as a $k$-majority tournament by $2 k-1$ ordered lists on $V$. McGarvey [3] showed that $k_{0}$ is well-defined for every $n$, and Erdös and L. Moser [2] showed $k_{0}(n)=O(n / \log n)$.

A dominating set in $T$ is a subset $W \subseteq V$, such that for every vertex $v \in V \backslash W$ there exists a vertex $u \in W$ such that $(u, v) \in E$. It was first demonstrated in [2], that there exist tournaments whose smallest dominating set is arbitrarily large, however, somewhat surprisingly, for $k$-majority tournaments that is not the case. In [1] it is shown that for $k$ majority tournaments the size of a smallest dominating set is bounded by a function of $k$, 


\begin{tabular}{|c|c|c|c|c|c|c|}
\hline \hline$k$ & 2 & 3 & 4 & 5 & 6 & 7 \\
\hline $\mathcal{F}(k)$ & 3 & 12 & 44 & 160 & 587 & 2177 \\
\hline
\end{tabular}

Table 1: Some upper bounds for small $k$

regardless of the size of the tournament, thus proving a conjecture of H.A. Kierstead and W.T. Trotter. For each $k$, let $\mathcal{F}(k)$ be the supremum of the size of a smallest dominating set in a $k$-majority tournament. Alon et al. [1] proved that $\mathcal{F}(2)=3$, that $\mathcal{F}(3) \geq 4$, and using results regarding the $\mathrm{VC}$ dimension of hypergraphs showed that $\mathcal{F}(k)=O(k \log k)$, and that $\mathcal{F}(k)=\Omega(k / \log k)$, for all $k$. However, the absolute constant for the former is shown in [1] to be larger than 80 .

The main results in this paper demonstrate upper bounds for $\mathcal{F}(k)$ which are exponential in $k$, but yield significantly improved bounds for small $k$, as demonstrated in Table 1. For instance, it is shown that $\mathcal{F}(3) \leq 12$. The upper bounds are achieved by using a skewed bipartite variation of the dominating set problem. This variation is also explored to some extent, and in addition, the main result provides an explicit proof to the conjecture of Kierstead and Trotter, as an alternative to the one given in [1] by other methods.

\section{$1.1 \quad$ Notation}

Given two distinct sets of vertices $A$ and $B$, we write $A \rightarrow B$ if $A$ dominates $B$, meaning for every $v \in B$ there exists $u \in A$ such that $(u, v) \in E$. Note that in a tournament it is always the case that $A \rightarrow B$ or $B \rightarrow A$. If $A=\{a\}$ and $A \rightarrow B$ we write $a \rightarrow B$, and vice versa. For a linear order $L$ on a set of vertices, let $L(u)$ denote the index of a vertex $u$. A vertex $u$ is said to beat a vertex $v$ in $L$ if $L(u)<L(v)(u$ is ranked higher than $v$ in $L$ ). Given a subset $A \subset V$ and a linear order $L$, the list induced by $A$ is obtained by deleting $V \backslash A$ from $L$, and retaining the order between the vertices of $A$.

\section{A variation on the dominating set problem}

Problem 1. Bipartite domination: Given two positive integers $x, y$, what is the smallest $h=h(x, y)$ such that for every tournament $T=(V, E)$, and partition $V=A \cup B$, for which $|A|=x,|B|=y$, there exists a subset $|C| \leq h$, such that $C \subseteq A$ and $C \rightarrow B$, or $C \subseteq B$ and $C \rightarrow A$ ?

Note that $h$ is well-defined as being dependent on $x, y$, as the number of tournaments of order $x+y$ is finite, and hence the number of partitions as above is finite, and it is always the case that either $A \rightarrow B$ or $B \rightarrow A$. Theorem 1 implies that the restriction of $h(x, y)$ to $k$-majority tournaments is bounded by a function of $k$ alone. Hence, we may denote by $F(k)$ the size of the smallest dominating set that is guaranteed to exist for the bipartite domination problem in $k$-majority tournaments, regardless of the size and 
partition of the vertex set. The following lemma demonstrates that the dominating set problem in a tournament is reducible to the bipartite domination problem.

Lemma 1. $\mathcal{F}(k) \leq F(k)$.

Proof. Let $T=(V, E)$ be a $k$-majority tournament. A 2-product of a tournament $T=$ $(V, E)$ is a tournament $T^{*}$ which is composed of two disjoint copies of $T$, such that if $v^{*}$ is a copy of $v$ and $u \in V, u \neq v$, then $\left(u, v^{*}\right) \in E\left(T^{*}\right)$ iff $(u, v) \in E$, else $\left(v^{*}, u\right) \in E\left(T^{*}\right)$. In addition, $\forall w \in V,\left(w, w^{*}\right) \in E\left(T^{*}\right)$. Let $T^{*}$ be the 2-product of $T$. Clearly, $k$ majority tournaments are closed under the 2-product construction, by inserting a copy of each vertex directly under it in each list. If $D$ is a solution to the bipartite domination problem in $T^{*}$, where the partition consists of the two distinct copies of $T$, then the sources of the vertices of $D$ in $V$ constitute a dominating set of $T$.

The following variant of majority tournaments will prove useful for generating bounds for the size of dominating sets.

Definition 1. A $(t, m)$-asymmetric bipartite majority tournament ( $A B M T), T=(V, E)$, consists of $m$ ordered lists on a set $V$, an integer $t$, such that $1 \leq t \leq m$, a disjoint ordered pair of sets $(A, B)$ satisfying $V=A \cup B$, and a set of edges $E$, such that for every $u \in A, v \in B,(u, v) \in E$ if and only if $u$ beats $v$ in at least $t$ lists, else $(v, u) \in E$.

Note that a $(t, m)$-ABMT given by $(A, B)$ and $L_{1}, \ldots, L_{m}$, is identical to the $(m-$ $t+1, m)$-ABMT given by $(B, A)$ and $L_{1}, \ldots, L_{m}$. The notion of an ABMT is useful if we know, for instance, that the vertices of a set $A$ beat all vertices of a set $B$ in specific lists, as in that case we may discard those lists and examine a certain ABMT in the remaining lists so that the edges between the vertices of the two sets are preserved.

The bipartite domination problem may be generalized in ABMT's. We will show by induction that the following proposition holds :

Proposition 1. There exists a function, $F^{*}: N \times N \rightarrow N$, such that for every $(t, m)$ $A B M T$ given by $(A, B)$ and $m$ lists, there exists a subset $C \subseteq A,|C| \leq F^{*}(t, m)$, and $C \rightarrow B$, or there exists a subset $D \subseteq B,|D| \leq F^{*}(m-t+1, m)$, and $D \rightarrow A$.

From Lemma 1 and the definition of $F(k)$ :

$$
\mathcal{F}(k) \leq F(k) \leq F^{*}(k, 2 k-1)
$$

At this stage we do not know yet whether $F^{*}$ can be defined, as for given $t, m$ there may be an infinite sequence of $(t, m)$-ABMT's for which $F^{*}(t, m)$ is unbounded. Note that the inequality in Equation 1 is due to the minimality of $F(k)$.

We will need the following definition for the inductive construction of the recurrence.

Definition 2. Let $T=(V, E)$ be a $(t, m)-A B M T$, given by lists $L_{1}, \ldots, L_{m}$, let $V=A \cup B$ be a partition of $V$, and let $1 \leq i \leq m$. The $(a, b)$ domination scheme of $(A, B)$ in $L_{i}$, is a pair $\left(\delta_{A}, \delta_{B}\right)$, such that $\delta_{A}$ and $\delta_{B}$ are the greatest indices in $L_{i}$ which satisfy: 

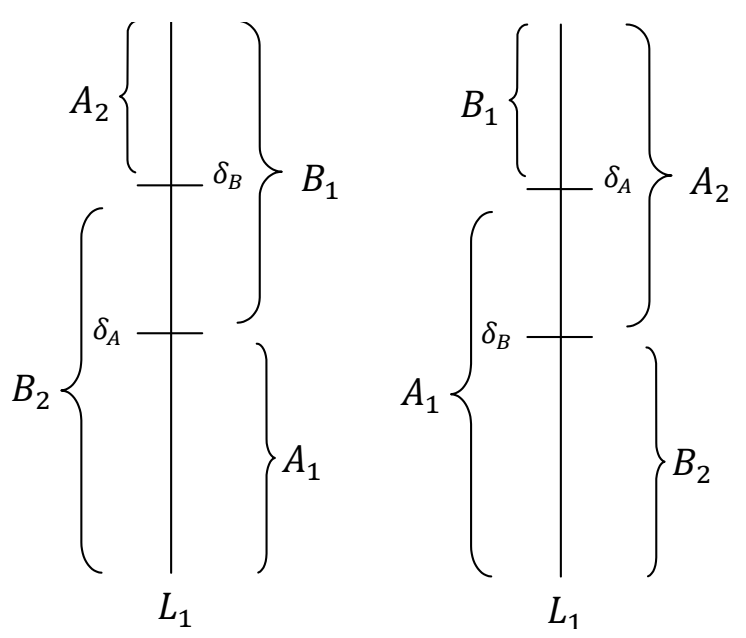

Figure 1: The two options for an $(a, b)$ domination scheme.

1. There exists a subset $C$ of $A$ satisfying $|C| \leq a$, such that $L_{i}(x) \geq \delta_{A}$ for every $x \in C$, and such that $C \rightarrow y$ whenever $y \in B$ satisfies $L_{i}(y)<\delta_{A}$.

2. There exists a subset $D$ of $B$ satisfying $|D| \leq b$, such that $L_{i}(x) \geq \delta_{B}$ for every $x \in D$, and such that $D \rightarrow y$ whenever $y \in A$ satisfies $L_{i}(y)<\delta_{B}$.

For any $i$, given the $(a, b)$ domination scheme of $(A, B)$ in $L_{i}$, we will use the following notation as in Figure 1: $B_{1}=\left\{x \in B, L_{i}(x)<\delta_{A}\right\}, A_{1}=\left\{x \in A, L_{i}(x) \geq \delta_{A}\right\}, A_{2}=$ $\left\{x \in A, L_{i}(x)<\delta_{B}\right\}, B_{2}=\left\{x \in B, L_{i}(x) \geq \delta_{B}\right\}$. Denote by $\Delta_{A}$ the union of all sets such as $C$ in definition 2 , and denote by $\Delta_{B}$ the union of all sets such as $D$. Note that in certain cases, such as a list where all vertices of $A$ beat all vertices of $B$ some of the defined sets may be empty, but it is not hard to check that in those cases the proofs to follow are simplified.

Lemma 2. Let $T=(V, E)$ be a $(t, m)-A B M T$ given by $(A, B)$ and lists $L_{1}, \ldots, L_{m}$, and let $\left(\delta_{A}, \delta_{B}\right)$ be the $(a, b)$ domination scheme of $(A, B)$ in $L_{i}$, for some $1 \leq i \leq m$. Assume w.l.o.g. that $\delta_{A} \geq \delta_{B}$ (as on the left in Figure 1). Denote by $v$ the vertex with index $\delta_{B}$. Then one of the following is true:

1. $v \in A, v \rightarrow \Delta_{B}$.

2. $v \in B, \delta_{B}<\delta_{A}, \Delta_{A} \rightarrow v$.

3. $v \in B, \delta_{B}=\delta_{A}, v \rightarrow \Delta_{A}$.

Proof. 1. If $v \in A$, then it cannot be the case that there is a vertex $u \in \Delta_{B}$ such that $u \rightarrow v$, otherwise, by definition, there is a subset $D \subseteq \Delta_{B}, u \in D$, and $D$ dominates all vertices of $A$ with index less or equal to $\delta_{B}$, and therefore $\delta_{B}$ should be greater than it is. 
2. If $v \notin A$ then $v \in B$. If $\delta_{B}<\delta_{A}$, then by definition there exists a set $C \subseteq \Delta_{A}$ such that $C \rightarrow v$.

3. If $v \in B$ and $\delta_{B}=\delta_{A}$, then in a similar fashion to case 1, it cannot be that there exists a vertex $w \in \Delta_{A}$ such that $w \rightarrow v$, and therefore $v \rightarrow \Delta_{A}$.

The main result that follows proves the existence of $F^{*}$ by showing that Proposition 1 holds for a class of functions that satisfy a certain recurrence.

Theorem 1. Let $F^{*}: N \times N \rightarrow N$ be a function for which:

1. $\forall m \geq 1, F^{*}(1, m)=m, F^{*}(m, m)=1$.

2. $\forall t>1, \forall m>t, F^{*}(t, m)=1+F^{*}(t, m-1)+F^{*}(t-1, m-1)$.

Then $F^{*}$ satisfies Proposition 1.

Proof. Let $T=(V, E)$ be a $(t, m)$-ABMT given by $(A, B)$ and lists $L_{1}, \ldots, L_{m}$.

1. If $t=1$ : Let $a_{i}$ be the element of $A$ with minimal index in $L_{i}, i=1 \ldots m$. If $\left\{a_{1}, \ldots, a_{m}\right\}$ does not dominate $B$, that means by the definition of $(t, m)$-ABMT's that there is an element $b \in B$ that does not have a greater index than $a_{i}$ in $L_{i}$, for all $1 \leq i \leq m$, and since $a_{i}$ is the minimal vertex of $A$ in each $L_{i}$, then $b$ beats all vertices of $A$ in all $m$ lists. Thus, we either have found a set of $m$ elements of $A$ that dominate $B$, or one element of $B$ that dominates $A$. The case $t=m$ is symmetric, and the same proof applies. Hence, for the cases $t=1$ or $t=m, F^{*}$ satisfies Proposition 1.

2. If $m>t>1$ : We will prove the result by induction on $m$. The basis of the induction is the case $m=3$, which will be resolved further on. Assume that the equality and Proposition 1 are true for all $m \leq x$, and let $m=x+1$. Let $\left(\delta_{A}, \delta_{B}\right)$ be the $\left(F^{*}(t, m-1), F^{*}(m-t+1, m-1)\right)$ domination scheme of $(A, B)$ in $L_{1}$. Denote by $v$ the vertex with index $\delta_{B}$ in $L_{1}$. Consider the following cases :

(a) $\delta_{A}>\delta_{B}$. (As on the left in Figure 1.) Note that in this case $B_{1} \cup B_{2}=B$.

i. If $v \in A$, then from case 1 in Lemma $2, v \rightarrow \Delta_{B}$.

There is a set $C_{A} \subseteq \Delta_{A},\left|C_{A}\right| \leq F^{*}(t, m-1)$, and $C_{A} \rightarrow B_{1}$. Let $L_{2}^{*}, \ldots, L_{m}^{*}$ be the induced lists given by $A_{2} \cup B_{2} \backslash \Delta_{B}$, and let $G$ be the $(t-1, m-1)$ ABMT given by $\left(A_{2}, B_{2} \backslash \Delta_{B}\right)$ and $L_{2}^{*}, \ldots, L_{m}^{*}$.

By the induction hypothesis, it must be the case that $A_{2} \rightarrow B_{2} \backslash \Delta_{B}$ in $G$ with at most $F^{*}(t-1, m-1)$ vertices, otherwise there is a subset $D_{B} \subseteq B_{2} \backslash \Delta_{B}$ of size at most $F^{*}(m-t+1, m-1)$ that dominates $A_{2}$ in $G$, however by the definition of $\Delta_{B}$, and since $\Delta_{B} \cap D_{B}=\emptyset$ that would be a contradiction, since if $D_{B} \rightarrow A_{2}$ in $G$, then $D_{B} \rightarrow A_{2}$ in $T$. (Because the domination of vertices in $A_{2}$ by vertices in $D_{B}$ takes place in $L_{2}^{*}, \ldots, L_{m}^{*}$, and therefore also in $L_{2}, \ldots, L_{m}$.) 
Therefore, there exists a subset $A_{3} \subseteq A_{2}$, such that $A_{3} \rightarrow B_{2} \backslash \Delta_{B}$ in $G$, and $\left|A_{3}\right| \leq F^{*}(t-1, m-1)$. Since all vertices of $A_{3}$ beat all vertices of $B_{2} \backslash \Delta_{B}$ in $L_{1}$, then $A_{3} \rightarrow B_{2} \backslash \Delta_{B}$ in $T$. Thus, $\{v\} \cup C_{A} \cup A_{3} \rightarrow \Delta_{B} \cup B_{1} \cup\left(B_{2} \backslash \Delta_{B}\right)=B$ in $T$, and :

$$
|\{v\}|+\left|C_{A}\right|+\left|A_{3}\right| \leq 1+F^{*}(t, m-1)+F^{*}(t-1, m-1) .
$$

ii. If $v \in B$, then from case 2 in Lemma $2, \Delta_{A} \rightarrow v$, meaning there is a vertex $w \in \Delta_{A}$ such that $w \rightarrow v$, and for some set $C_{A}, w \in C_{A} \subseteq \Delta_{A}, C_{A} \rightarrow B_{1}$, and $\left|C_{A}\right| \leq F^{*}(t, m-1)$.

Since $v \in B_{2}$, the only reason why $\delta_{B}$ is not greater than it is, can be that there is no set $D_{B} \subseteq \Delta_{B} \backslash\{v\}, D_{B} \leq F^{*}(m-t+1, m-1)$, and $D_{B} \rightarrow A_{2}$. Define $G$ as the $(t-1, m-1)$-ABMT given by $\left(A_{2}, B_{2} \backslash\{v\}\right)$ and the induced lists $L_{2}^{*}, \ldots, L_{m}^{*}$. It must be that $A_{2} \rightarrow B_{2} \backslash\{v\}$ in $G$ with at most $F^{*}(t-1, m-1)$ vertices, or there is a set of size $F^{*}(m-t+1, m-1)$ in $B_{2} \backslash\{v\}$ that dominates $A_{2}$ in $G$, and therefore in $T$, contradicting the inexistence of $D_{B}$. Let $A_{3} \subseteq A_{2}$ be the set such that $A_{3} \rightarrow B_{2} \backslash\{v\}$ in $G$ (and therefore in $T$ as in case 2(a)i), and $\left|A_{3}\right| \leq F^{*}(t-1, m-1)$. Thus, $C_{A} \cup A_{3} \rightarrow B_{1} \cup B_{2}=B$, and :

$$
\left|C_{A}\right|+\left|A_{3}\right| \leq F^{*}(t, m-1)+F^{*}(t-1, m-1) .
$$

(b) $\delta_{A}<\delta_{B}$. (As on the right in Figure 1.) Following similar arguments as in case 2 a, while $v$ is assigned to be the vertex with index $\delta_{A}$, results in a set $D_{B},\left|D_{B}\right| \leq F^{*}(m-t+1, m-1)$, such that $D_{B} \rightarrow A_{2}$, and if $v \in B$, then $v \rightarrow \Delta_{A}$, and $B_{1} \rightarrow A_{1} \backslash \Delta_{A}$ with at most $F^{*}(m-t, m-1)$ vertices in the $(m-t, m-1)$-ABMT given by $\left(B_{1}, A_{1} \backslash \Delta_{A}\right)$ in the induced lists $L_{2}^{*}, \ldots, L_{m}^{*}$, or in the case that $v \in A, B_{1} \rightarrow A_{1} \backslash\{v\}$ with at most $F^{*}(m-t, m-1)$ vertices in the $(m-t, m-1)$-ABMT given by $\left(B_{1}, A_{1} \backslash\{v\}\right)$ in the induced lists $L_{2}^{*}, \ldots, L_{m}^{*}$. Thus, the case $v \in B$ is the worse of the two, therefore $A$ is dominated in $T$ by a subset of $B$ of size at most :

$$
|\{v\}|+\left|D_{B}\right|+F^{*}(m-t, m-1) \leq 1+F^{*}(m-t+1, m-1)+F^{*}(m-t, m-1) .
$$

(c) $\delta_{A}=\delta_{B}$. In this case, as shown in cases 3, 1 in Lemma 2, either $v \in A$ and $v \rightarrow \Delta_{B}$, or $v \in B$ and $v \rightarrow \Delta_{A}$. In the first case we follow case $2($ a)i, and in the second case we follow case $2 \mathrm{~b}$ when $v \in B$.

(d) The inductive basis is for $m=3$. In this case the (2,3)-ABMT is a 2majority tournament in which we solve the bipartite domination problem. By $1, F^{*}(2,2)=1$, meaning we are employing a $(1,1)$ domination scheme, therefore due to symmetry we can assume $\delta_{A}>\delta_{B}$, and by following case $2(\mathrm{a}) \mathrm{i}$ and plugging $m=3$ we obtain $F^{*}(2,3)=1+F^{*}(2,2)+F^{*}(1,2)=4$.

Given a $(t, m)$-ABMT, for $m>t>1$, we have shown that there either exists a subset $C \subseteq A, C \rightarrow B,|C| \leq 1+F^{*}(t, m-1)+F^{*}(t-1, m-1)=F^{*}(t, m)$, or there exists $D \subseteq B, D \rightarrow A,|D| \leq 1+F^{*}(m-t+1, m-1)+F^{*}(m-t, m-1)=F^{*}(m-t+1, m)$, as required. 
Combining 1 and 2 , the result holds for all $m \geq t \geq 1$, and therefore for all $(t, m)$-ABMT's. Hence, $F^{*}$ satisfies Proposition 1.

\section{$3 \quad$ Upper bounds for $\mathcal{F}(k)$}

The results provide upper bounds for $\mathcal{F}(k)$ which are exponential in $k$. However, for small $k$ (see Table 1 ), these bounds are rather small. We may already use Theorem 1 to generate bounds for $\mathcal{F}(k)$, but the following result reduces $F^{*}(2, m)$ by $m-2$, and $F^{*}(m-1, m)$ by 1 . (Those values may be easily calculated from Theorem 1 to be $\frac{m(m+1)}{2}-2,2 m-2$ respectively.)

Theorem 2. Let $F^{*}: N \times N \rightarrow N$ be a function for which :

1. $\forall m \geq 1, F^{*}(1, m)=m, F^{*}(m, m)=1$.

2. $\forall m \geq 3, F^{*}(2, m)=\frac{m(m-1)}{2}, F^{*}(m-1, m)=2 m-3$

3. $\forall t>2, \forall m>t+1, F^{*}(t, m)=1+F^{*}(t, m-1)+F^{*}(t-1, m-1)$.

Then $F^{*}$ satisfies Proposition 1.

Proof. The only change in $F^{*}$ is for $t=2, m-1$. We prove the result by induction on $m$. Let $T=(V, E)$ be a $(2, m)$-ABMT given by $(A, B)$ and lists $L_{1}, \ldots, L_{m}$. As in Theorem 1 , by plugging $t=2,\left(\delta_{A}, \delta_{B}\right)$ is the $\left(F^{*}(2, m-1), F^{*}(m-1, m-1)\right)$ domination scheme of $(A, B)$ in $L_{1}$. By Theorem $1,\left(\delta_{A}, \delta_{B}\right)$ is a $\left(F^{*}(2, m-1), 1\right)$ domination scheme.

The improvement is obtained by a transitivity argument. Assume $\delta_{A}>\delta_{B}$. As similar to Theorem 1 , if $v \in A$, where $v$ is the vertex with index $\delta_{B}$ in $L_{1}$, then $v \rightarrow \Delta_{B}$. In addition there exists a set $C_{A} \subseteq \Delta_{A},\left|C_{A}\right| \leq F^{*}(2, m-1)$, and $C_{A} \rightarrow B_{1}$. Let $L_{2}^{*}, \ldots, L_{m}^{*}$ be the induced lists given by $A_{2} \cup B_{2} \backslash \Delta_{B}$. Denote by $G$ the $(1, m-1)$-ABMT given by $\left(A_{2}, B_{2} \backslash \Delta_{B}\right)$ and $L_{2}^{*}, \ldots, L_{m}^{*}$. As in Theorem 1 , there exists a subset $A_{3} \subseteq A_{2}$, such that $A_{3} \rightarrow B_{2} \backslash \Delta_{B}$ in $G$, and $\left|A_{3}\right| \leq F^{*}(1, m-1)=m-1$.

As in case 1 in Theorem 1 , the vertices of $A_{3}$ that dominate $B_{2} \backslash \Delta_{B}$ in $G$ may be taken as $\left\{a_{2}, \ldots, a_{m}\right\}$, being the vertices of $A_{2}$ with minimal index in $L_{2}, \ldots, L_{m}$, and since $\Delta_{B} \rightarrow A_{2}$ in $T$, then every element of $A_{2}$ is beaten by each element of $\Delta_{B}$ in all the lists $L_{2}, \ldots, L_{m}$. (Since $\Delta_{B}$ consists of vertices that participate in a set of size 1 that dominates $A_{2}$ in $T$.) $v \rightarrow \Delta_{B}$ in $T$, so $v$ has a smaller index than every element in $\Delta_{B}$ in at least one of the lists $\left\{L_{2}, \ldots, L_{m}\right\}$ (in addition to $L_{1}$ ), and therefore a smaller index than $a_{i}$ in $L_{i}$, for some $2 \leq i \leq m$. By transitivity, $v$ dominates all vertices of $B_{2} \backslash \Delta_{B}$ that $a_{i}$ dominates in $L_{1}, L_{i}$, so $v$ may replace $a_{i}$ in the set that dominates $B_{2} \backslash \Delta_{B}$ in $T$. Denote by $A_{3}=\left\{a_{2}, \ldots, a_{i-1}, v, a_{i+1}, \ldots, a_{m}\right\}$. Since all vertices of $A_{2}$ and $v$ have a smaller index than $B_{2} \backslash \Delta_{B}$ in $L_{1}$, then $A_{3} \rightarrow B_{2} \backslash \Delta_{B}$ in $T$. Therefore, since $v \rightarrow \Delta_{B}$, then $A_{3} \cup C_{A} \rightarrow B_{1} \cup\left(B_{2} \backslash \Delta_{B}\right) \cup \Delta_{B}=B$. Therefore :

$$
\left|A_{3}\right|+\left|C_{A}\right| \leq m-1+F^{*}(2, m-1)=m-1+\frac{(m-1)(m-2)}{2}=\frac{(m-1)(m-2)+2(m-1)}{2}=\frac{m(m-1)}{2} .
$$


If $v \in B$, then following the same arguments as in the parallel case in Theorem 1 , we define $G$ as the $(1, m-1)$-ABMT given by $\left(A_{2}, B_{2} \backslash\{v\}\right)$ and the induced lists $L_{2}^{*}, \ldots, L_{m}^{*}$, resulting in $C_{A} \cup A_{3} \rightarrow B$ in $T$ (in this case $A_{3}=\left\{a_{2}, \ldots, a_{i}, \ldots, a_{m}\right\}$ ). Thus :

$$
\left|A_{3}\right|+\left|C_{A}\right| \leq F^{*}(1, m-1)+F^{*}(2, m-1)=m-1+F^{*}(2, m-1)=\frac{m(m-1)}{2} .
$$

If $\delta_{A}<\delta_{B}$ then, as in Theorem 1 , by plugging $t=2$ and using the induction hypothesis, $A$ is dominated in $T$ by a subset of $B$ of size at most :

$1+F^{*}(m-1, m-1)+F^{*}(m-2, m-1)=2+F^{*}(m-2, m-1)=2+2(m-1)-3=2 m-3$.

The case $\delta_{A}=\delta_{B}$ is dealt with as in Theorem 1 . The induction base is for $m=3$, for which we compute the value of $F^{*}(2,3)$. As in case $2 \mathrm{~d}$ in Theorem 1 , due to symmetry, w.l.o.g. we may follow the case $\delta_{A}>\delta_{B}$ as above, and produce a dominating set of size $\frac{3 \times 2}{2}=3$, which validates the induction basis and improves the previous value by 1 .

Thus, given a $(2, m)$-ABMT defined by $(A, B)$ and lists $L_{1}, \ldots, L_{m}$, we have shown that there exists either a subset of $A$ of size at most $\frac{m(m-1)}{2}$ that dominates $B$, or a subset of $B$ of size at most $2 m-3$ that dominates $A$, as required.

It is interesting to note that for 2-majority tournaments, the result in [1] produces a dominating set of size at most three, however, given a partition of the vertex set $V=A \cup B$, it may be that of the dominating set two vertices are in $A$ and one in $B$. The following corollary shows that the dominating set may always be chosen to be contained either in $A$ or in $B$.

Corollary 1. $F(2)=3$.

Proof. From Equation 1, Lemma 1, and the fact that $\mathcal{F}(2)=3$ :

$3=\mathcal{F}(2) \leq F(2) \leq F^{*}(2,3)=3$.

It is now easy to see how to compute bounds for $\mathcal{F}(k)$, as the following Corollary demonstrates.

Corollary 2. $\mathcal{F}(3) \leq 12$.

Proof. From Equation 1, $\mathcal{F}(3) \leq F(3) \leq F^{*}(3,5)$. Therefore, by Theorem 2 :

$\mathcal{F}(3) \leq F^{*}(3,5)=1+F^{*}(3,4)+F^{*}(2,4)=1+(2 \times 4-3)+\frac{4 \times 3}{2}=1+5+6=12$.

It may be possible to obtain a closed form inequality for our upper bound for $\mathcal{F}(k)$, however, for small $k$, one obtains the bounds listed in Table 1 using a straightforward computer program.

\section{Further remarks}

1. Theorem 4 in [1] shows an upper bound of $O(k \log (k))$ for $\mathcal{F}(k)$ by using a two-person zero-sum game on the set of vertices, such that the sum of incoming probabilities on each vertex is at least $\frac{1}{2}$. It is possible to design a game on a bipartite tournament with similar qualities to prove a similar bound for $F(k)$. 
2. It is now known that $4 \leq \mathcal{F}(3) \leq 12$. It may be possible to reduce the gap by checking sufficiently large random 3-majority tournaments.

3. It is not a difficult exercise to construct a 2-majority tournament with a partition of its vertex set in to sets of equal size such that both parts dominate each other, but only one side of the partition dominates the other with $F(2)=3$ vertices, while the other side needs all its vertices in order to dominate.

\section{Acknowledgment}

The author thanks Raphael Yuster for introducing him to the problem and advice on editing of this paper, and thanks to the referees for useful comments.

\section{References}

[1] N. Alon, G. Brightwell, H. A. Kierstead, A. V. Kostochka, P. Winkler, Dominating sets in k-majority tournaments, J. Combinatorial Theory, Ser. B 96 (2006), 374-387.

[2] P. Erdös and L. Moser, On the representation of directed graphs as unions of orderings, Magyar Tud. Akad. Mat. Kutató Int. Közl. 9 (1964), 125-132.

[3] D.C. McGarvey, A theorem on the construction of voting paradoxes, Econometrica 21(1953), 608-610. 